\title{
Transient-thermo-reflectance for the study of surface carrier dynamics
}

\section{Reflectancia-termo-transitoria para el estudio de la dinámica de portadores de carga en superficies}

\author{
B.G. Pérez-Hernández(A), J. Garduño-Mejía ${ }^{(*, A)}$, C. J. Román-Moreno( ${ }^{(A)}$, \\ O. G. Morales-Saavedra, J. G. Bañuelos-Muñetón, R. Ortega-Martínez ${ }^{(\mathrm{A})}$ \\ CCADET-UNAM, Apdo, Postal 70-186, C.P. 04510. Coyoacán, Cd. Universitaria, México D.F., México. \\ (*) Email: jesus.garduno@ccadet.unam.mx A: miembro de AMO / AMO member \\ Recibido / Received: 06/09/2012. Revisado / Revised: 13/12/2012. Aceptado / Accepted: 18/12/2012. \\ DOI: http://dx.doi.org/10.7149/OPA.46.1.13
}

\begin{abstract}
:
In this work we present a non-destructive examination (NDE) pump-probe technique known as transient-thermo-reflectance (TTR) for the study of transient carrier dynamics in semiconductor and metal surfaces at low fluence regime. The technique enables to measure the change in reflectance at the sample surface as a function of time on the femtosecond time regime. Changes in reflectance can then be used to determine properties of the sample. Experimental results are compared with numerical model calculations.
\end{abstract}

Key words: Ultrafast Optics, Femtosecond Time Resolved Spectroscopy.

\section{RESUMEN:}

En este trabajo presentamos una técnica no destructiva de bombeo-prueba, técnica conocida como reflectancia termo-transitoria (TTR) para el estudio de la dinámica de portadores de carga en superficies metálicas y semiconductoras a un régimen de fluencia baja. La técnica permite medir el cambio en la reflectancia en la superficie de la muestra como función del tiempo en el régimen temporal de femtosegundos. Los cambios en la reflectancia pueden emplearse para determinar las propiedades de la muestra. Los resultados experimentales se comparan con los cálculos de un modelo numérico.

Palabras clave: Óptica Ultrarrápida, Espectroscopia de Resolución Temporal de Femtosegundos.

\section{REFERENCIAS Y ENLACES / REFERENCES AND LINKS}

[1]. P. M. Norris, A. P. Caffrey, R. J. Stevens, J. M. Klopf, J. T. McLeskey Jr., A. N. Smith, “Femtosecond pumpprobe nondestructive examination of materials", Rev. Sci. Instrum. 74, 400-406 (2003). DOI

[2]. J. Garduño-Mejía, M. P. Higlett, S. R. Meech, "Modelling the influence of nonthermal electron dynamics in thin and ultrathin gold films", Chem. Phys. 341, 276-284 (2007). DOI

[3]. J. Garduño-Mejía, M. P. Higlett, S. R. Meech, "Morphology dependent ultrafast electron dynamics in ultrathin gold films", Surf. Sci. 602, 3125-3130 (2008). DOI

[4]. R. H. M. Groenveld, R. Sprink, A. Lagendijk, "Femtosecond spectroscopy of electron-electron and electron-phonon energy relaxation in Ag and Au", Phys. Rev. B 51, 11433-11445 (1995).

[5]. C. K. Sun, F. Vallee, L. H. Acioli, E. P. Ippen, J. G. Fujimoto, "Femtosecond tunable measurement of electron thermalization in gold", Phys. Rev. B 50, 15337-15348 (1994). DOI

[6]. B. N. Chichkov, C. Momma, S. Nolte, F. von Alvensleben, A. Tünnermann, "Femtosecond, picosecond and nanosecond laser ablation of solids", Appl. Phys. A - Mater. 63, 109-115 (1996). DOI

[7]. S. I. Anisimov, B. L. Kapeliovich, T. L. Perelman, "Electron emission from metal surfaces exposed to ultrashort laser pulses", Soviet Physics - JETP 39, 375-377 (1974). 
[8]. E. Carpene, "Ultrafast laser irradiation of metals: Beyond the two-temperature model", Phys. Rev. B 74, 024301 (2006). DOI

[9]. J. Hohlfeld, S. S. Wellershoff, J. Gudde, U. Conrad, V. Jahnke, E. Matthias, “Electron and lattice dynamics following optical excitation of metals", Chem. Phys. 251, 237-258 (2000). DOI

[10].S. K. Sundaram, E. Mazur, "Inducing and probing non-thermal transitions in semiconductors using femtosecond laser pulses", Nat. Mater. 1, 217-224 (2002). DOI

[11]. R. P. Prasankumar, A. J. Taylor, Optical Tecniques for Solid-State Materials Characterization, CRC Press, Taylor and Francis Group, Boca Raton, FL, USA (2012).

[12]. F. Rossi, T. Kuhn, "Theory of ultrafast phenomena in photoexcited semiconductors", Rev. Mod. Phys. 74, 895-950 (2002). DOI

\section{Introduction}

Ultrashort pulsed lasers have been demonstrated to be effective tools for the nondestructive examination (NDE) of energy transport properties in thin film samples, in particular for the investigation of transient dynamics as well as nonlinear optical properties in semiconductors and metals. A good alternative for the NDE pump-probe technique is known as transient-thermo-reflectance (TTR) for the study of transient dynamics in semiconductor and metal materials [1]. The technique enables to measure the change in reflectance at the sample surface as a function of time on a sub-picosecond time scale. Changes in reflectance can then be used to determine properties of the sample. In the case of metals, the change in reflectance is related to changes in temperature and strain. The transient temperature profile at the surface is then used to estimates the rate of energy transfer and coupling factor between the electron and phonon as well as the thermal properties of the material [1-9]. In the case of semiconductors, the change in the reflectance is related to local electronic state changes and temperature [10-12].

\section{Experimental setup}

In TTR technique a femtosecond pulse is split into an intense heating pulse and a weaker probe pulse (Fig. 1). The heating pulse is used to generate the transient event to be observed. Control of the optical path length of the probe pulse produces a variable time delay between the pump and probe pulses. The probe then takes a snapshot of the reflectance at a specific experimental time delay relative to the pump, with a temporal resolution on the order of the probe pulse duration. In our case the delay line is capable of producing time steps of 3 fs. Pulses

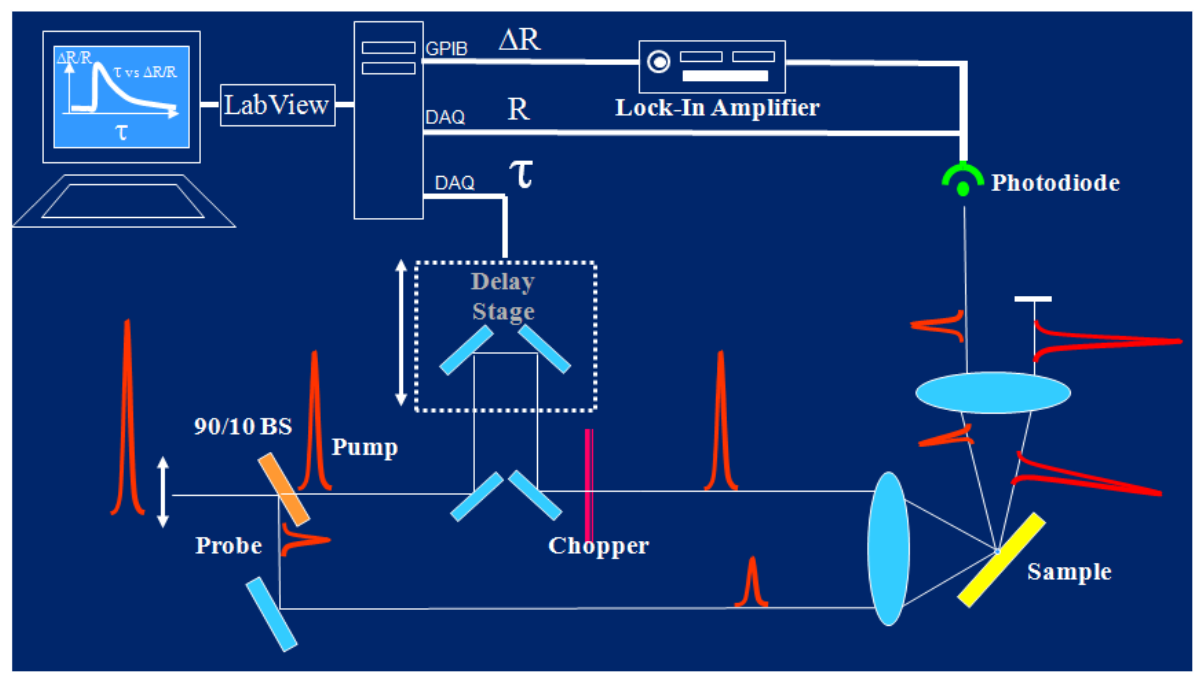

Fig. 1. Transient-thermo-reflectance experimental setup. 
from a self-mode-locked femtosecond Ti:sapphire laser delivering $200 \mathrm{fs}, 76 \mathrm{MHz}$, centered at $810 \mathrm{~nm}$ (Coherent MIRA-900) were divided in the pump and probe with a wedged window. Typical energy per pulse is about $1.5 \mathrm{~nJ}$. The pump beam passes through a chopper, creating a modulated pulse train at a prescribed frequency of $2 \mathrm{KHz}$, which will drive the reference frequency and phase of a lock-in amplifier.

The non collinear pump and probe beams, with P polarization, are focused to a spot size of about $4 \mu \mathrm{m}$ with a $25 \mathrm{~mm}$ focal length lens at an incident angle of $45^{\circ}$ from the normal. With this setup the transient reflectance can be associated with carrier dynamics in both metals and semiconductors.

Since the method is non-invasive, it represents a good alternative for measurements of thermal properties on thin-layer materials.

\section{Results}

Time resolved spectroscopy results using the TTR technique are presented in bulk GaAs and a $5 \mathrm{~nm}$ thickness Au sample deposited on a glass substrate in the low fluence excitation regime. We divided the analysis for the metal and the semiconductor.

\subsection{Metal}

Due to the lattice structure of metals, the electrons are not localized to particular atoms but in a gas-like dispersed throughout the metal. The occupancy, at a particular energy state, is governed by the Fermi-Dirac distribution. Energy transfer in metals can be described as follows: after a femtosecond laser pulse excitation, electrons are promoted to highly nonequilibrium states. Following the excitation, the electron thermalization is established by collisions between excited electrons and electrons around the Fermi level. Once thermal equilibrium among hot electrons is reached its temperature differs strongly from the lattice temperature, due the large heat capacity difference, and then diffusion processes and electron-phonon coupling takes place and cools down the electron bath. Finally electrons and lattice reach thermal equilibrium.
Diffusive electron motion and cooling by electron-phonon coupling can be well described by the two-temperature model (TTM) and solved numerically to predict the time and spatial dependence ( $t$ and $z$ ) of electron $\left(T_{e}\right)$ and lattice temperature $\left(T_{l}\right)[1-3,6-9]$ :

$$
\begin{aligned}
C_{e}\left(T_{e}\right) \frac{\partial T_{e}}{\partial t} & =\frac{\partial}{\partial z}\left(K_{e}\left(T_{e}, T_{l}\right) \frac{\partial T_{e}}{\partial z}\right)- \\
& -g_{e-p h}\left[T_{e}-T_{l}\right]+S(z, t), \\
C_{l} \frac{\partial T_{l}}{\partial t} & =g_{e-p h}\left[T_{e}-T_{l}\right]
\end{aligned}
$$

where the source term is:

$$
S(z, t)=0.94 J \frac{1-R}{\tau_{p} \delta} \exp \left[-\frac{z}{\delta}-2.77\left(\frac{t}{\tau_{p}}\right)^{2}\right]
$$

The model is based on coupled electronlattice heat transfer differential equations (Eqs. (1) and (2)) and source term (Eq. (3)) which involve different optical properties, such as the reflectance of the film, $R$, excitation source parameters and others described in Table I $[2,4,8,9]$.

Transient optical response is estimated by the computation of the corresponding transient dielectric function for a given wavelength. The dielectric function depends directly on the Fermi-Dirac distribution function, which depends on the time-dependent electron temperature, and density of states evolution (DOS) of the material [2].

The TTM has been demonstrated to be consistent in bulk samples but shows some differences for thin films, below $10 \mathrm{~nm}$. For

Table I

Optical, excitation and thermal parameters of the $\mathrm{Au}$.

\begin{tabular}{|c|l|l|}
\hline$g_{e-p}$ & $\begin{array}{l}\text { Electron-phonon } \\
\text { coupling factor }\end{array}$ & $2.67 \times 10^{16} \mathrm{~W} / \mathrm{Km}^{3 *}$ \\
\hline$C_{e}=A_{e} T_{e}$ & $\begin{array}{l}\text { Electron heat } \\
\text { capacity }\end{array}$ & $A_{e}=67.96 \mathrm{~J} / \mathrm{m}^{3} \mathrm{~K}$ \\
\hline$C_{l}$ & Lattice heat capacity & $2.5 \times 10^{6} \mathrm{~J} / \mathrm{m}^{3} \mathrm{~K}$ \\
\hline$K_{e}$ & $\begin{array}{l}\text { Electron thermal } \\
\text { conductivity }\end{array}$ & $318 \mathrm{~W} / \mathrm{mK}$ \\
\hline$J$ & Laser fluence & $36 \mathrm{~J} / \mathrm{m}^{2}$ \\
\hline$\tau_{p}$ & Pulse width & $200 \mathrm{fs}$ \\
\hline$\delta$ & $\begin{array}{l}\text { Optical penetration } \\
\text { depth }\end{array}$ & $70 \mathrm{~nm}$ \\
\hline
\end{tabular}

${ }^{*}$ Bulk value 
instance, energy rate transfer between electrons and lattice is governed by the electron-phonon coupling parameter, $g_{e-p h}$, which is characteristic for each material. Some investigations have suggested that this parameter could depend on the film thickness and structure [3].

In Fig. 2 the TTR for a $5 \mathrm{~nm} \mathrm{Au}$ film, fabricated by evaporation, is presented with corresponding pump excitation fluence of 36 $\mathrm{J} / \mathrm{m}^{2}$.

A drawback of the TTM is that it assumes instantaneous thermalization so that the actual behavior in the first stage after the excitation, in this case after 2 ps, is not described correctly. In order to compare the transient behavior just during the decaying stage in Fig. 2, the TTM numerical result was shifted in time to match the normalized maximum of the experimental measurement.

In the bulk sample, on the other hand, hot electrons diffuse into greater depths with the electron-phonon coupling governing the diffusion length. This diffusion leads to the fast, almost exponential relaxation. On the other hand, in thin samples, the hot electron diffusion is inhibited and the energy is stored in a much smaller volume [9]. The electrons cool exclusively by coupling to the lattice, resulting in a near-linear decay of the electron temperature. This near-linear behaviour is observed in our results.

The reported ge-ph parameter for bulk or a continuous film of $\mathrm{Au}$ corresponds to $2.67 \times 10^{16}$ $\mathrm{WK}^{-1} \mathrm{~m}^{-3}[2-4]$. In order to get a better fit with our experimental data we reduced this parameter to $2 \times 10^{16} \mathrm{WK}^{-1} \mathrm{~m}^{-3}$ in the TTM, according to previous reported work [3]. The reduction of the coupling electron-phonon parameter is translated to a decrement in the energy transfer rate. In Fig. 2 we present the fit for the two different values of $g_{e-p h}$.

It has been reported previously that the morphology of metal films changes from continuous to particulate as the mass thickness is decreased below a few tens of nanometers [3]. This result confirms that the formation of particles in thin metal films affects the effectiveness of energy transfer between electron and lattice.

The presence of particle formation was confirmed with UV-VIS absorption measurements with a particle plasmon resonance shift to about $575 \mathrm{~nm}$.

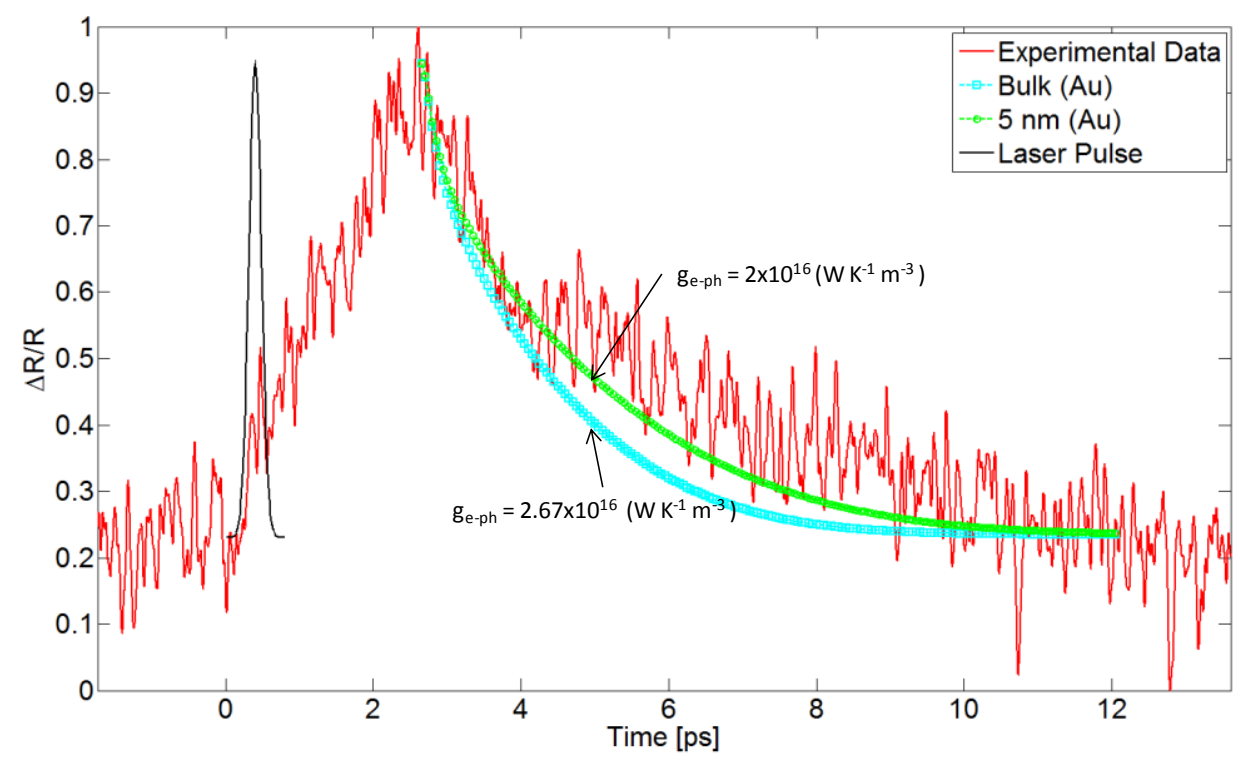

Fig. 2: Normalized experimental transient-thermo-reflectance $(\Delta R / R)$ on $5 \mathrm{~nm}$ of Au (solid red), femtosecond laser pulse (solid black) and the two-temperature-model (TTM) fitting for two different electron-phonon coupling parameter $g_{e-p h}$ (symbols green and cyan). 


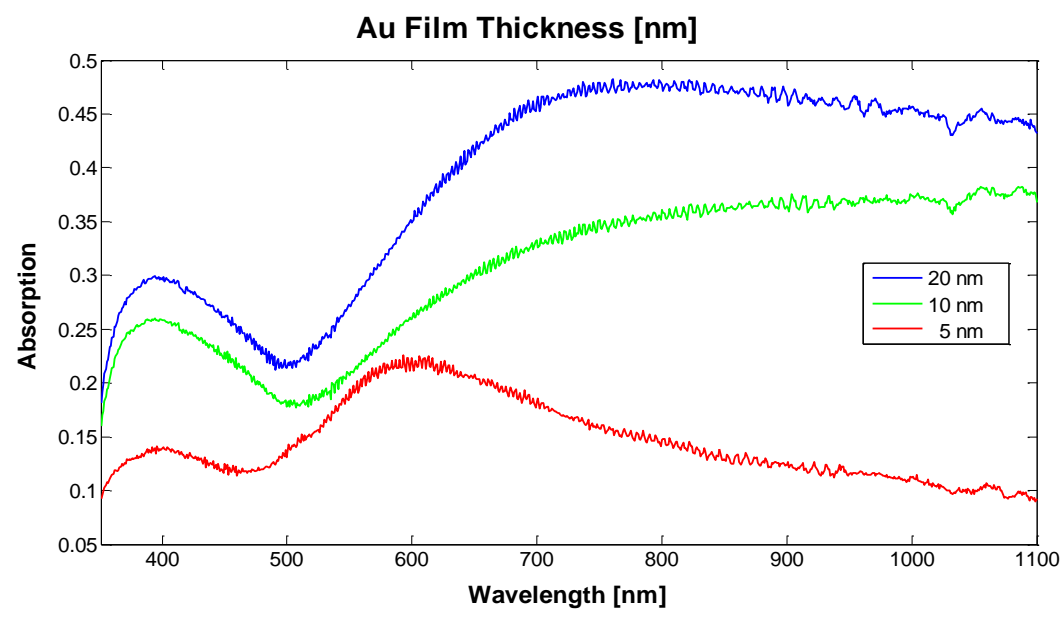

Fig. 3: UV-VIS absorption data for 5, 10 and $20 \mathrm{~nm}$ Au films deposited on a glass surface.

The absorption peak shift is demonstrated in Fig. 3 where the absorption spectra for 3 different film thicknesses are presented: 5 (TTR experimental sample), 10 and $20 \mathrm{~nm}$. Actual TTR experimental results for $\mathrm{Au}$ films with thicknesses of $10 \mathrm{~nm}$ and $20 \mathrm{~nm}$ have been presented previously $[1,2]$.

The presence of some structure was also confirmed with atomic force microscopy measurements presented in Fig. 4.

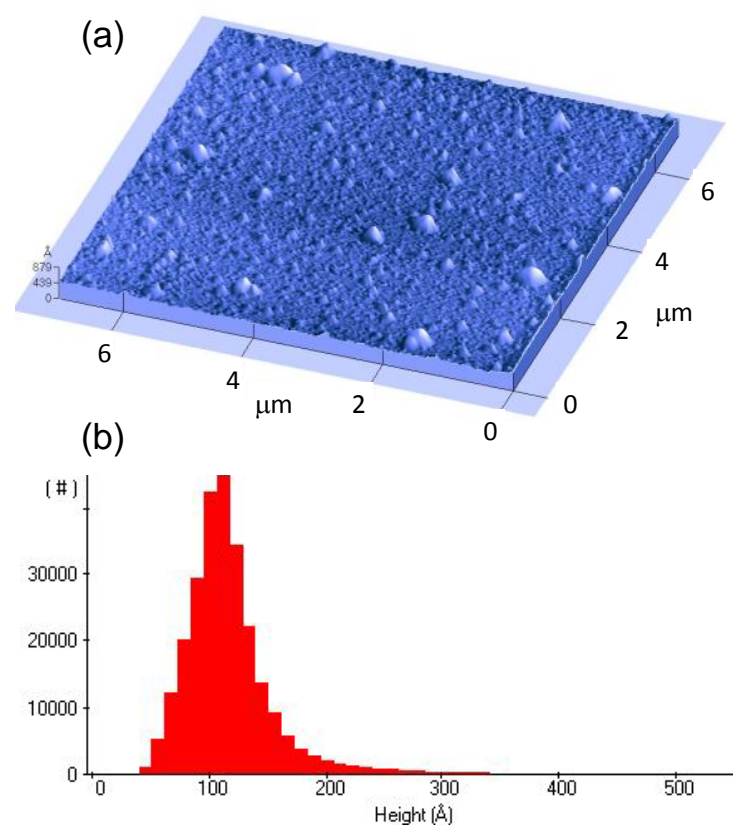

Fig. 4: AMF image of the $5 \mathrm{~nm} \mathrm{Au}$ sample (a) and height histogram (b).

\subsection{Semiconductor}

On excitation with an ultrashort pulse, a semiconductor undergoes several stages of relaxation before returning to equilibrium [10-12]. The energy is transferred first to the electrons and then to the lattice. The interaction includes several regimes of carrier excitation and relaxation as a function of time.

Table II summarizes the hierarchy of the different regimes as a function of time including the most relevant interactions $[10,11]$.

Figure 5 illustrates some of the processes that take place in the regimes of Table I for a typical direct-gap semiconductor. The various processes shown do not occur sequentially; they

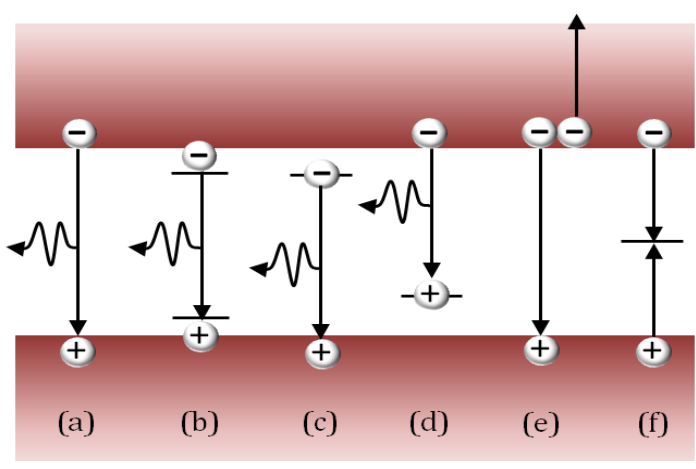

Fig. 5: Schematic sketch of different recombination processes in bulk semiconductors: (a) band-to-band or direct recombination, (b) exciton recombination, (c) donator-band recombination, (d) acceptor-band recombination, (e) Auger recombination, (f) recombination via deep levels. (a) through d) are radiative recombination processes, where a photon is emitted. (e) and (f) are non-radiative recombination channels. 
Table II

Overview of different temporal regimes of a semiconductor after excitation with an ultrashort laser pulse.

\author{
Regime and Timescale \\ Optical excitation 10-100 \\ fs
}

Coherent regime 10 fs to several 100 fs

\author{
Nonthermal regime $<200$ \\ fs
}

\section{Thermalized or hot- carrier regime 100 fs to ps}

\section{Recombination or} isothermal regime ps to $\mu \mathrm{s}$

\section{Relevant Interaction}

Optical dipole moment of interband transitions (or intersubband transitions in quantum structures)

Interaction of optical polarization with electronic states, decay of coherent polarization through scattering processes

Carrier-carrier scattering

Carrier-carrier scattering Carrier-phonon interactions
Radiative or non-radiative recombination

Carrier trapping

\section{Remarks}

The duration of the excitation pulse is closely related to the energetic width of the excited carrier distribution

Description in terms of coherent wave functions, coherent polarizations, and optical Bloch equations, observation of coherent wavepacket dynamics (quantum beats, Bloch oscillations)

Carrier distribution cannot be assigned a temperature, occurrence of a spectral hole

Carrier distribution is defined by a temperature; temperatures of electron and hole distributions may be different and are larger than lattice temperature; temperatures of electron and hole distributions equilibrate with the lattice temperatures

Temperatures of carrier distributions and lattice are equal; timescale strongly depends on the material, e.g., direct or indirect semiconductors, density of defects, and quality of surfaces

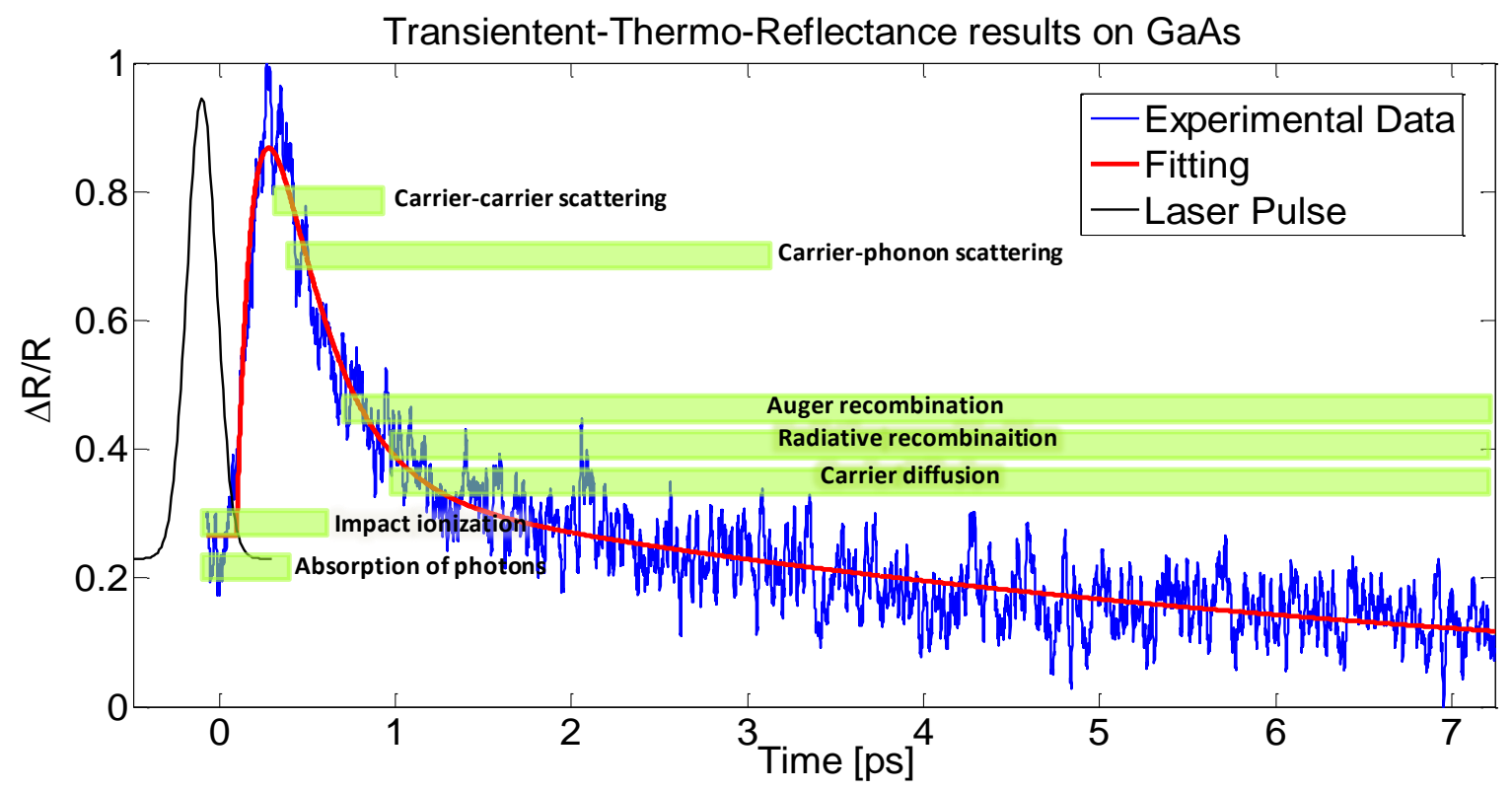

Fig. 6: Transient-thermo-reflectance response of GaAs (solid blue) and the femtosecond laser pulse (solid black). 
overlap in time, forming a continuous chain of events spanning the entire range from femtoseconds to microseconds. For example, carriers thermalize at the same time as they cool by transferring energy to lattice phonons. Nonthermal structural effects (for example formation of transient structures) can occur while the lattice is still cold.

In Fig. 6 we present the TTR technique applied to GaAs with the description of different processes which take place at different times, achieved at an excitation fluence of $36 \mathrm{~J} / \mathrm{m}^{2}$.

It has been reported in previous works that below $0.5 \mathrm{~kJ} / \mathrm{m}^{2}$, or about $50 \%$ of the threshold for irreversible structural change $\left(F_{t h}\right)$, only laser-induced lattice heating is produced [10]. With less than one order of magnitude below this fluence level we were able to generate a transient thermo reflectance response directly associated with the thermalization time or hotcarrier regime. The experimental result was fitted to the following Eq. (4):

$$
\begin{aligned}
R & =A\left(1-\exp \left[-\frac{\tau}{t_{s}}\right]\right) \exp \left[-\frac{\tau}{t_{R_{1}}}\right]+ \\
& +B\left(1-\exp \left[-\frac{\tau}{t_{R_{1}}}\right]\right) \exp \left[-\frac{\tau}{t_{R_{2}}}\right]+ \\
& +C\left(1-\exp \left[-\frac{\tau}{t_{R_{2}}}\right]\right),
\end{aligned}
$$

where $\tau$ is the time delay, $t_{s}$ is the rise time, $t_{R_{1}}$ and $t_{R_{2}}$ are the relaxation times, $A, B, C$ are amplitude parameters and $R$ is the fitting result.

Fitting time parameters are listed in Table III.

\section{Conclusions}

Preliminary results based on femtosecond timeresolved spectroscopy demonstrates transient behaviour of the reflectance at the excitation focal point, which can be related to the change of
Table III

Fitting time parameters from TTR experimental results on GaAs.

\begin{tabular}{|c|c|c|c|}
\hline $\begin{array}{c}\text { Fluence } \\
{\left[\mathrm{J} / \mathrm{m}^{2}\right]}\end{array}$ & $t_{s}[\mathrm{ps}]$ & $t_{R_{1}}[\mathrm{ps}]$ & $t_{R_{2}}[\mathrm{ps}]$ \\
\hline 36 & 0.32 & 0.24 & 16.27 \\
\hline
\end{tabular}

population of excited carriers on both semiconductor and metal surfaces. Nondestructive examination (NDE) of energy transport technique has been demonstrated in the low fluence regime of $36 \mathrm{~J} / \mathrm{m} 2$ with $\mathrm{Au}$ and GaAs. In the case of the Au sample, transient temperature, heat transfer parameters and coupling factor related to energy transfer rate between electrons and phonons were corroborated with a numerical model. Comparison between numerical and experimental results suggest that the formation of particles in thin Au films, below $10 \mathrm{~nm}$, affects the effectiveness of energy transfer between electron and lattice. The nano-structured surface was demonstrated with UV-VIS spectroscopy and AFM measurements. For the case of GaAs, at very low fluence regime, one tenth of the threshold for irreversible structural changes, we have demonstrated a transient response with relaxation times between 240 fs to 16 ps. As far as we know this is the lowest excitation fluence that has been reported to achieve transient thermo reflectance for both metals and semiconductors.

\section{Acknowledgements}

The authors gratefully acknowledge the support of Dr. M. E. Mata-Zamora for the UV-VIS spectral measurements and the sponsorship of this work by DGAPA-UNAM. PAPIIT projects: IN113809, IB101212, IACOD-1100611, BID-UNAM 98-B3-CDGP-L0034-1077 and CONACyT-Mexico project CB 131746. 